\title{
APPLICATIONS OF A NODAL-INTEGRATION-BASED FINITE ELEMENT METHOD TO NON-LINEAR PROBLEMS
}

\author{
Yabo.Jia $^{1}$, Jean-Baptiste.Leblond ${ }^{2}$, Jean-Christophe.Roux ${ }^{1}$, Remi.Lacroix ${ }^{3}$ and \\ Jean-Michel.Bergheau ${ }^{1}$ \\ ${ }^{1}$ University of Lyon, ENISE, LTDS, CNRS, UMR 5513. \\ 58 rue Jean Parot, 42023 Saint-Etienne Cedex 02, France, \\ yabo.jia@enise.fr, jean-christophe.roux@enise.fr, jean-michel.bergheau@enise.fr \\ ${ }^{2}$ University of Sorbonne, Institut Jean Le Rond dAlembert, CNRS, UMR7190 \\ 4 place Jussieu, 75005 Paris, France \\ jbl@1mm.jussieu.fr \\ ${ }^{3}$ ESI FRANCE, batiment Le Rcamier, 70 rue Robert, 69006 Lyon, France
}

Key words: Nodal integration technique, Tetrahedral meshes, Numerical simulation, Bending, Elastoplasticity, volumetric locking, Thermo-mechanical simulation

\begin{abstract}
In this paper, we firstly introduce a nodal-integration-based finite element method. The method allows the use of first-order tetrahedral elements without suffering from the volumetric locking problem. The most important advantage of tetrahedral meshes is that they can be automatically generated for complex geometries using existing reliable meshing tools. The method is then applied to 3 types of applications. The first application is a large displacement, large strains elastic-plastic simulation on a notched specimen. The second application is an elastic-plastic bending problem. And the last example concerns the numerical simulation of the thermo-mechanical problem. In all the cases, the solution given by the nodal-integration-based FEM is compared to more classical FEM results.
\end{abstract}

\section{INTRODUCTION}

Finite element simulations of the behavior of structures made up of materials obeying the von Mises plasticity criterion (the most commonly used criterion especially for metal materials) face particular difficulties. These difficulties come from the plastic incompressibility condition imposed by the behavior of the material. This condition of (nearly-)incompressibility which should be resolved at each integration point can lead to a volumetric locking phenomenon.

Finite element formulations have thus been proposed to alleviate these difficulties. These formulations are generally based either on reduced numerical integration schemes, or on mixed formulations of the problem $[1,2]$. In practice, formulations based on reduced integration schemes are easily applicable only with hexahedral elements. The absence of automatic meshing tools with this type of finite element then leads engineers to tedious operations which are costly in human time. The existence of automatic tetrahedral meshing tools gives this type of finite element an indisputable economic advantage. New 
first order tetrahedral finite elements have thus been developed in recent years on the basis of mixed displacement-pressure formulations of the problem $[3,4,5]$. However, the presence of different degrees of freedom (displacement and pressure) or even that of internal degrees of freedom for certain elements, such as the element $\mathrm{P} 1+\mathrm{P} 1[6,7]$, can then lead to significant additional computation costs.

In the literature, choosing the nodes as integration points can be found both in meshless methods and in the finite element method. In meshless methods especially, the Stabilized Conforming Nodal Integration (SCNI) technique proposed by Chen et al. $[8,9,10]$ performs very well in several benchmark problems. The principle of SCNI technique is to consider nodal deformations calculated on average over a volume surrounding the node. The volume integral is then transformed into a surface integral thanks to the divergence theorem (Figure 1). These volumes can be obtained for example from a tesselation of Voronoi cell. Puso et al. [11] has proposed a schema of improvement for solving low energy mode that appears in the nodal integration technique.

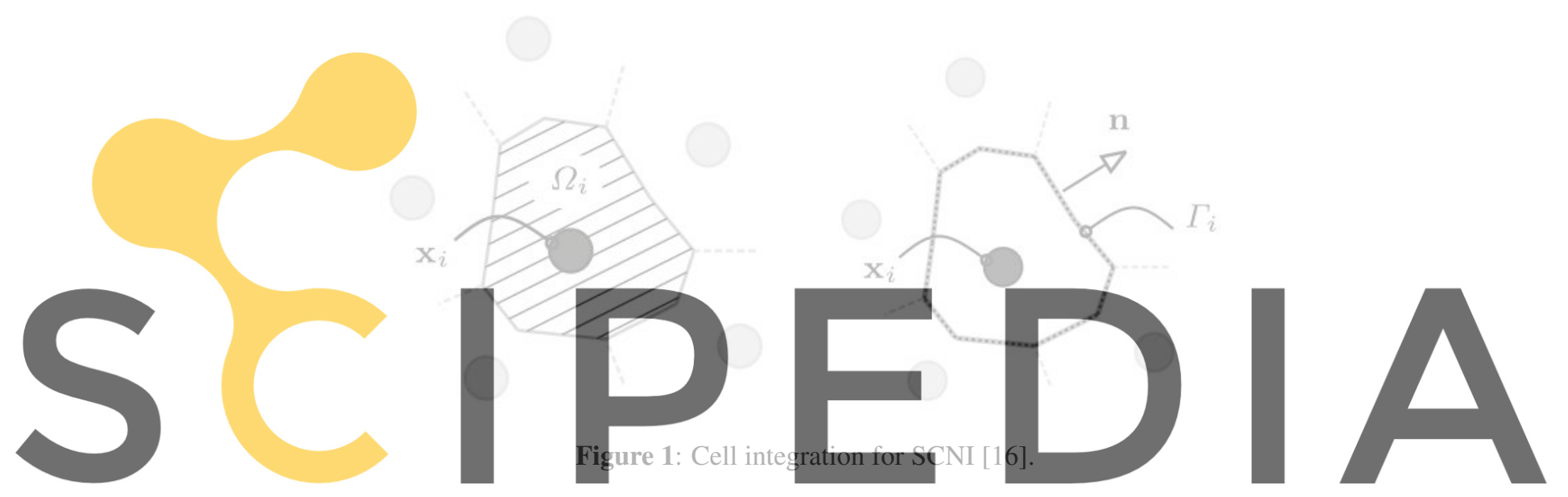

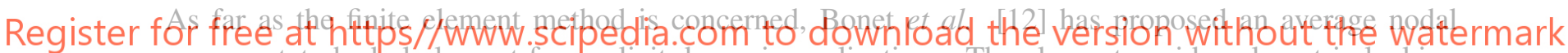
pressure tetrahedral element for explicit dynamic applications. The element avoids volumetric locking problems by computing average nodal pressures from surrounding elements. Dohrmann et al. [13] et al. presented a new tetrahedral element for small strain elasticity with an averaging procedure now applied on all the strain components. The formulation prevents the volumetric locking by producing a favorable constraint ratio for the volumetric response. Bonnet et al. [14] has extended this node-based formulation to explicit dynamics applications. Krysl et al. [15] presents assumed-strain finite elements based on nodal integration. They show that the three-node triangle, four-node tetrahedron, and eight-node and 27-node hexahedra give good performance in both compressible and almost incompressible regime. The combined use of the Finite Element Method with the SCNI technique has been developed in 2D on the basis of triangular meshes by Qual et al.[16] and Canales et al. [17]. Ce method has been extended for 3D thermo-mechanical applications by Jia et al. [18]. In this paper, we present several benchmark tests by comparing the results obtained with the nodal-integration-based finite element method as proposed by Jia et al. [18] with those coming from more classical finite element solutions. All the simulations are performed using SYSWELD ${ }^{T M}$ software. 


\section{Notched tensile specimen}

The objective of the first test is to compare the nodal-integration-based solution to classical FEM solutions on a 3D large strain elastoplastic problem. For symmetry reasons, only half of the specimen on an angular area is considered. Symmetry boundary conditions are applied to the faces of the model. The numerical model is presented in figure 2. Four numerical simulations based on the following formulations have been performed and compared:

1. P1 element: linear variation of the displacements inside the element,

2. P1P1 element: linear variation of the displacements and pressure inside the element

3. P1+P1 element: linear variation of the displacements and pressure inside the element plus 3 additional internal degrees of freedom associated with a bubble shape function.
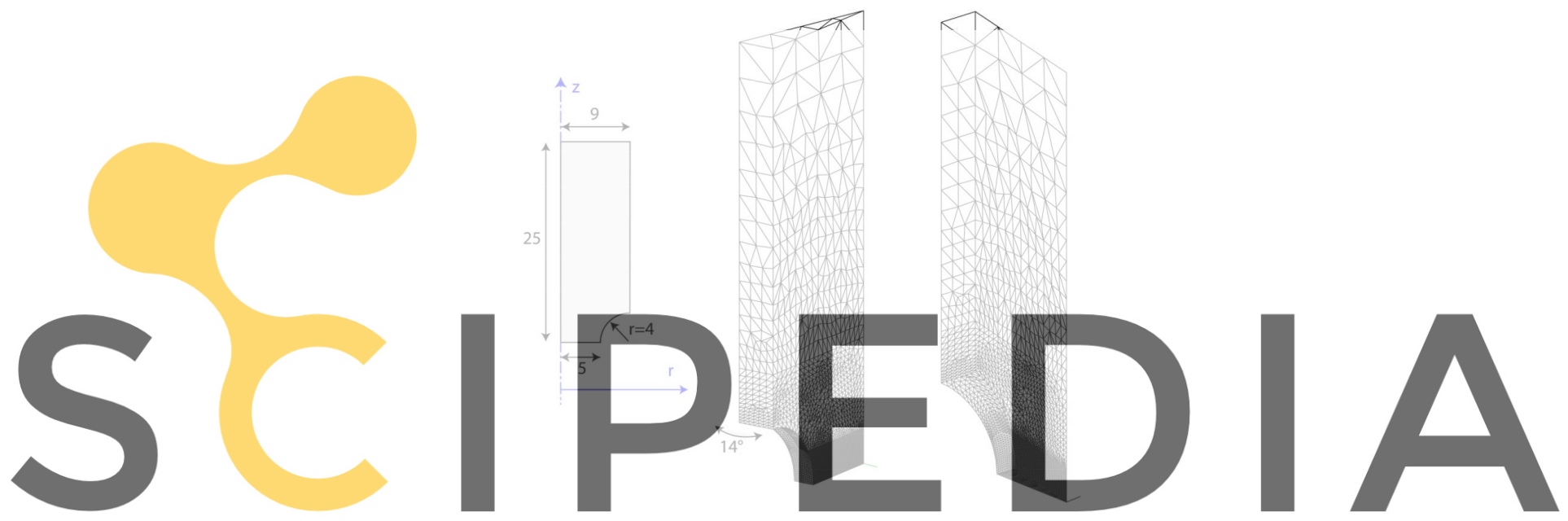

Figure 2: Mesh of the notched tensile specimen (unit: $\mathrm{mm}$ ).

\section{Register for free at https//www.scipedia.com to download the version without the watermark}

Large displacements, large strains are taken into account with an updated lagrangian formulation. The steel behavior is supposed to be elastoplastic with an isotropic hardening. The Young's modulus $E=$ $200,000 \mathrm{MPa}$, the Poisson ratio $v=0.3$ and the yield stress is a function of the cumulated equivalent plastic strain $\sigma_{y}\left(\varepsilon_{e q}^{p}\right)=\mathrm{A}\left(\varepsilon_{e q}^{p}+\varepsilon_{e q 0}^{p}\right)^{n}$ with $A=500 \mathrm{MPa}, n=0.1$ and $\varepsilon_{e q 0}^{p}=0.01$. Initial yield stress $\sigma_{y 0}=\mathrm{A}\left(\varepsilon_{e q 0}^{p}\right)^{n}=315.48 \mathrm{MPa}$.

Figure 3 provides comparisons of force-axial displacement curves and CPU times. P1 element is known to be too stiff and to present volumetric locking problems. As expected it gives a result far from those from the other formulations. The nodal-integration-based FEM leads to a force-axial displacement curve very close to those obtained using $\mathrm{P} 1 \mathrm{P} 1$ and $\mathrm{P} 1+\mathrm{P} 1$ elements, without any volumetric locking problem while requiring less CPU time.

Figure 4 shows the axial displacement and axial stress distributions obtained with P1+P1 element and the nodal-integration-based FEM. The displacement and stress distributions are very close. 


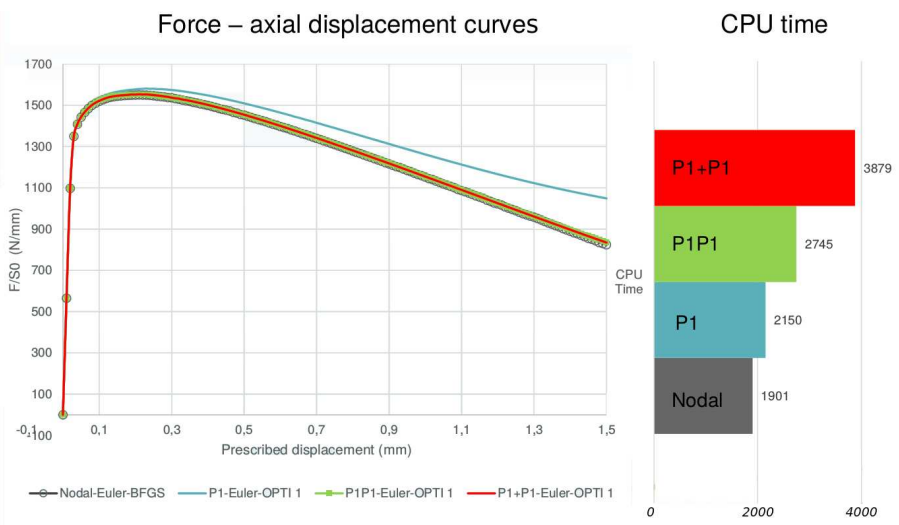

Figure 3: Force-axial displacement curves and CPU times.

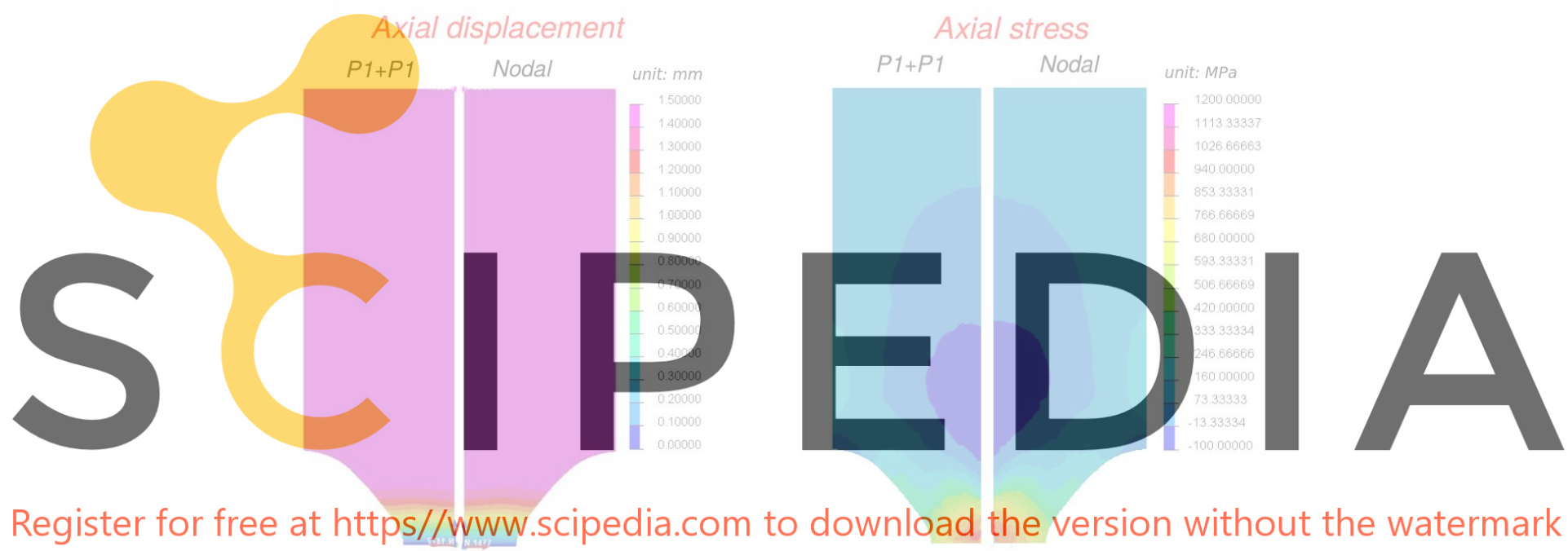

Figure 4: Stresses given by nodal-integration-based FEM and PI+PI element.

\section{Large deformation elastoplastic bending}

As we know, the tetrahedral P1 element exhibits poor performances in bending dominated problems. Therefore the present section is dedicated to the investigation of bending performances of different approaches. We consider a beam on simple support at its ends and loaded in the middle. For symmetry reasons, only a quarter of the beam is considered. Figure 5 shows the model including loading and boundary conditions and the meshes considered. Mesh (a) is prepared for Q2 hexahedral elements. It is well known that Q2 elements give very accurate results for bending problems [1,2]; the Q2 element solution will be therefore considered as a reference solution. Mesh (b) is prepared for Q1P0 hexahedral elements based on a mixed displacement (Q1)-pressure (P0) formulation. Mesh (c) is prepared for linear tetrahedral elements (P1P1 element, nodal-integration-based FEM). The dimensions of the model are $1 * 2 * 12 \mathrm{~mm}$. An elastoplastic behavior law is considered with isotropic hardening and a constant 

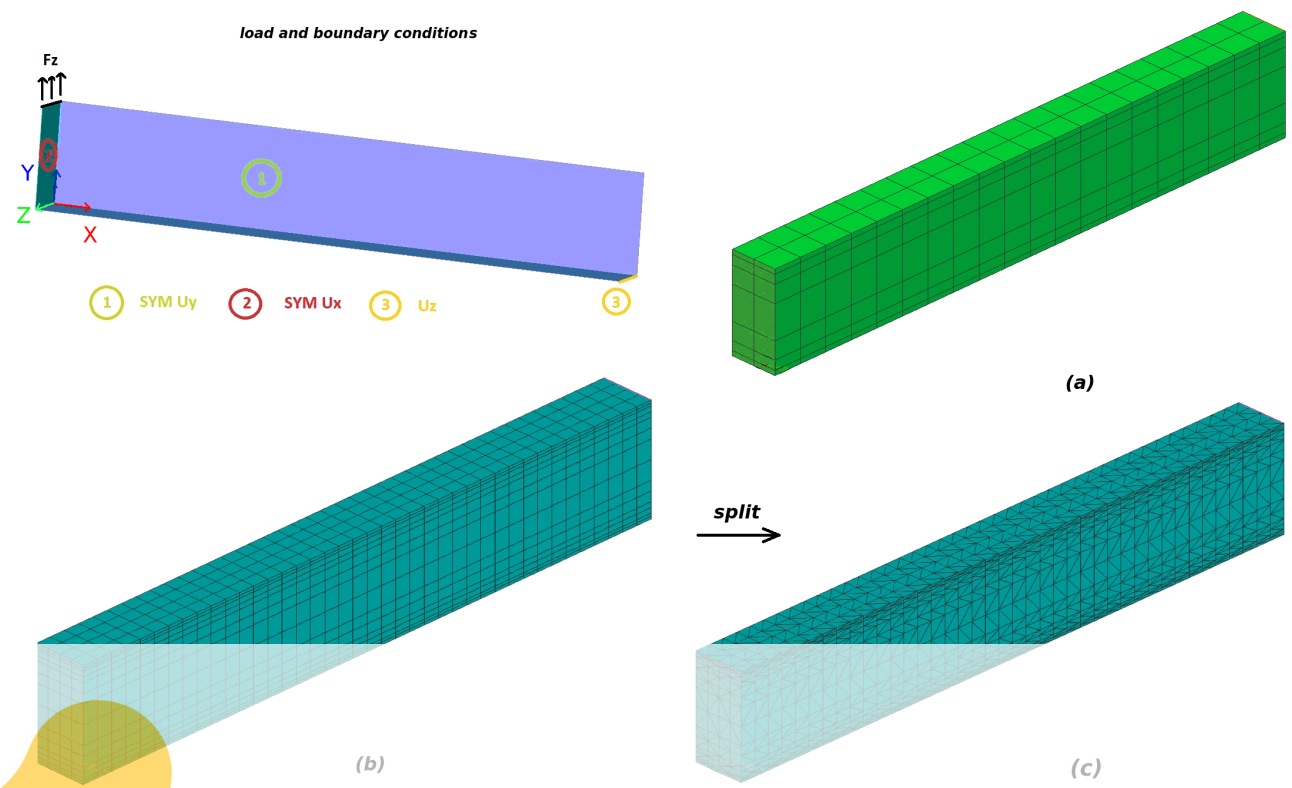

Figure 5: Numerical model and boundary conditions for bending simulations; meshes-(a) hexahedral mesh: 1,721 nodes/ 280 Q2 elements; (b) hexahedral mesh: 3,075 nods/ 2,240 Q1 elements; (c) tetrahedral mesh: 3075 nodes/ 13,440 tetrahedral elements.
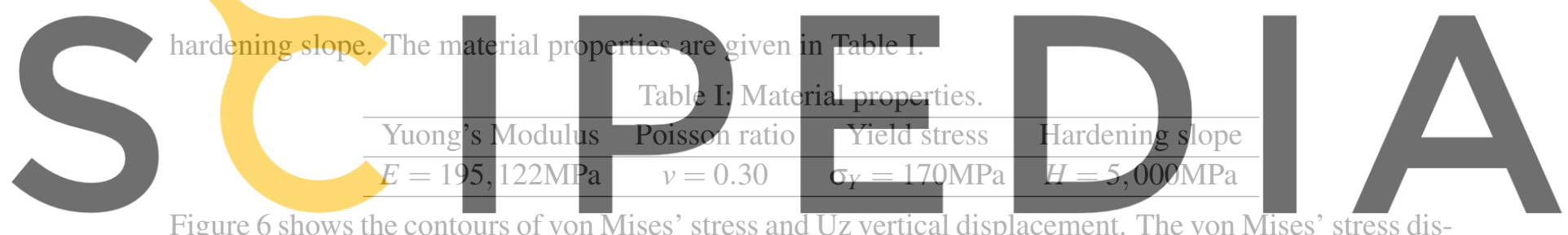

Figure 6 shows the contours of von Mises' stress and Uz vertical displacement. The von Mises' stress dis-

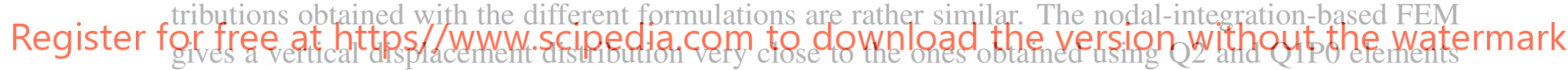

while the P1P1 element exhibits a too stiff bending behavior. This comes from the fact that because in-

tegration points are the nodes of the mesh, the nodal-integration-based FEMI leads to an underintegration of the problem.

Table II provides a comparison of the maximum vertical displacement and of the CPU times obtained with the different approaches. Compared with the reference solution (Q2 element), the nodal-integrationbased FEM exhibits a behavior a little too flexible. But, despite the increase of the matrix bandwidth it leads [18], the nodal-integration-based FEM gives a CPU time significantly less than the ones obtained with Q1P0 and P1P1 elements for a problem gathering the same number of nodes. This is related to the elastoplastic resolutions that are performed at the elements integration points with the Q1P0 and P1P1 meshes and at the nodes (which are much less numerous than the integrations points) with the nodal-integration-based FEM. 
Table II : Maximum displacement in $\mathrm{Z}$ direction.

\begin{tabular}{cccccc}
\hline element type & integration point & $U_{z}$ & $\Delta z$ & $\frac{\Delta z}{\left|U_{z}\right|}$ & CPU \\
\hline Q2 element & 5600 & -3.95 & reference & reference & 187 \\
Q1P0 element & 17920 & -3.88 & -0.07 & -0.0177 & 578 \\
P1P1 element & 13440 & -3.38 & -0.57 & -0.144 & 860 \\
nodal-integration-based FEM & 3075 & -4.11 & 0.16 & 0.04 & 256 \\
\hline
\end{tabular}
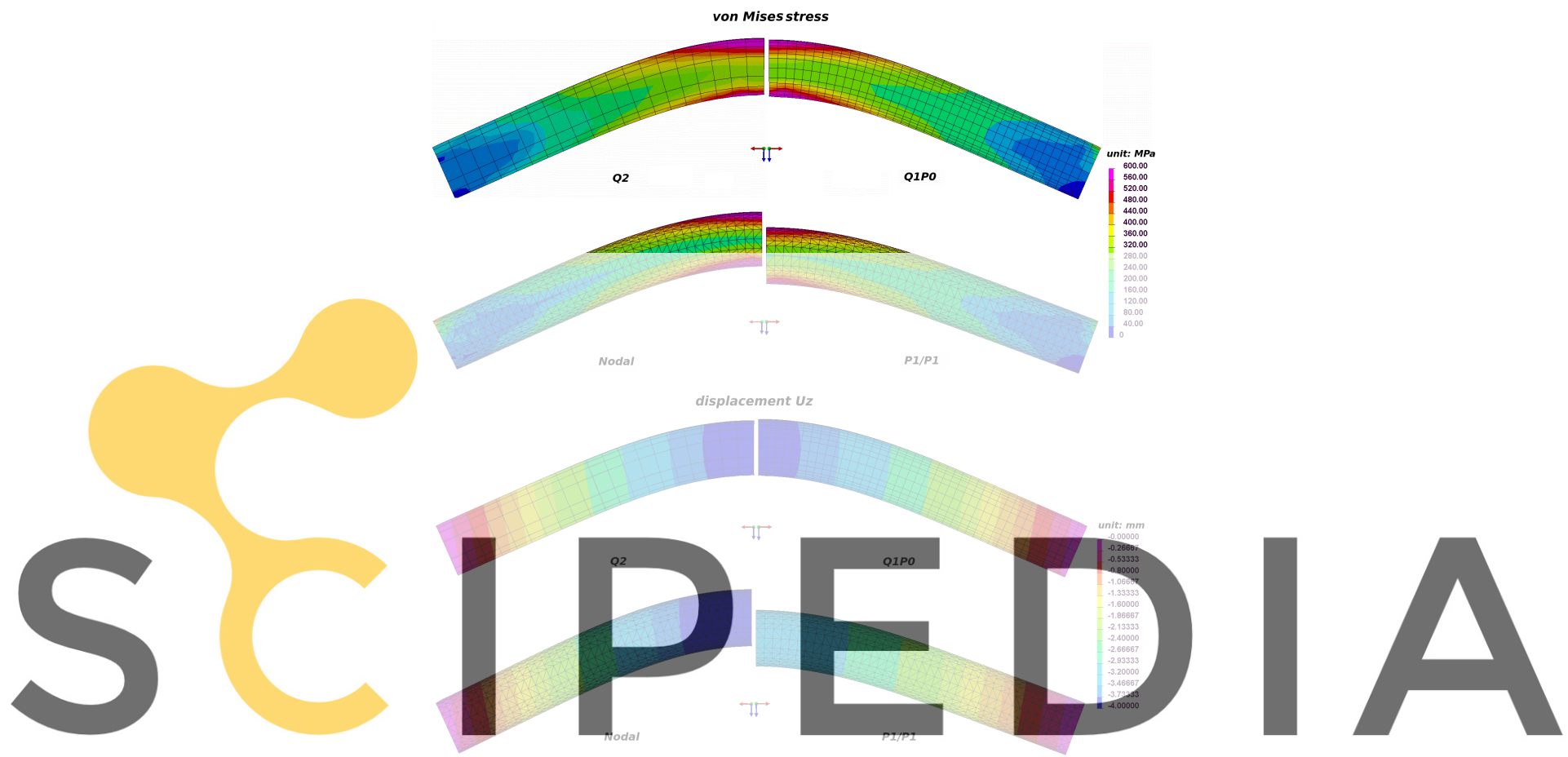

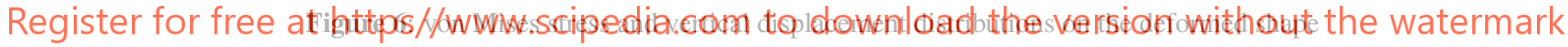

\section{Thermo-mechanical simulation}

In this section, we will present the application of thermo-mechanical simulation. The dimensions of problems and meshes are shown in figure 7 . This problem is solved by performing a $2 \mathrm{D}$ axisymmetry simulation. A gaussian heat source is applied and its energy distribution is described by equation (1). The heat source parameters are $a=8, b=2$, and $Q_{0}$ which is given in Table II. The center of the heat source is defined at $\left(X_{0}=0, Y_{0}=5\right)$. $316 \mathrm{~L}$ material properties taken from SYSWELD ${ }^{T M}$ database [19] is used in this simulation. The convective and radiative losses are taken into account.

$$
Q_{(X, Y)}=Q_{0}(t) * S Q R T\left(1-\frac{\left(X-X_{0}\right)^{2}}{a^{2}}-\frac{\left(Y-Y_{0}\right)^{2}}{b^{2}}\right)
$$


Table II: $Q_{0}$ in function of time.

\begin{tabular}{ccccc}
\hline time (second) & 0 & 1.5 & 3 & 100 \\
\hline$Q_{0}$ & 0 & 20 & 0 & 0
\end{tabular}

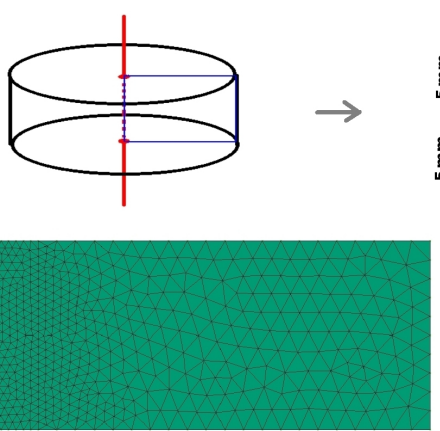

(a)
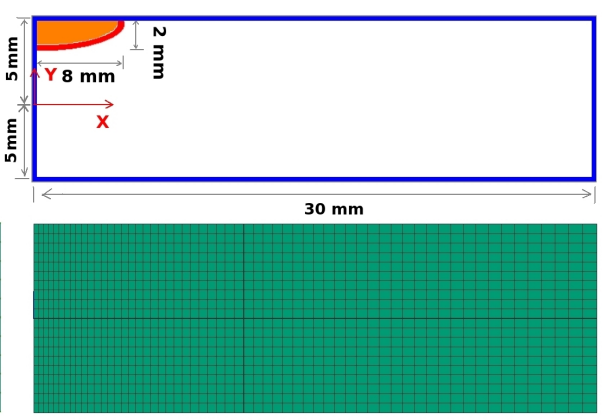

(b)

Figure 7: Problem studied and meshes - (a) triangle mesh: 942 nodes / 1849 2D elements; (b) quadrilateral mesh: 1281 nodes/1200 2D elements.

Figure 8 shows the residual stresses given by the nodal-integration-based FEM and classical FEM and the contours of residual stresses show no difference.

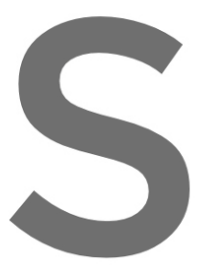

radial stress $\left(\sigma_{-}\right.$rr $)$(unit: MPa)
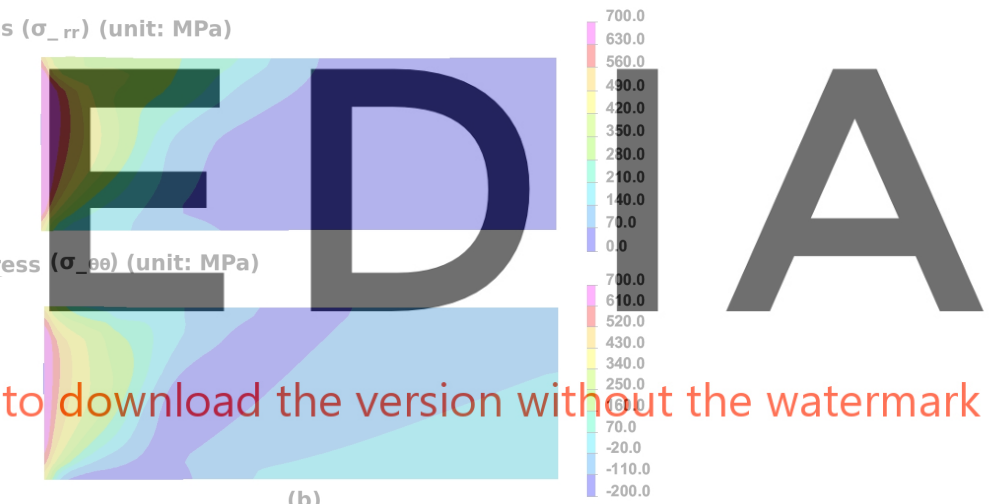

(b)

Figure 8: Residual stresses simulated by (a): nodal-integration-based FEM and (b): Q1 FEM.

\section{CONCLUSIONS}

Three applications of the nodal-integration-based finite element method have been presented. These applications are representative of different classical problems: large strain elastoplasticity, bending dominated problem, thermo-mechanical application. All the simulation are carried out with the same PC.

According to the comparisons, the nodal-integration-based FEM gives accurate results. The main advantage of the method lies on the use of tetrahedral meshes. There is no longer any volumetric locking problem. Meshing of complex structures is so greatly facilitated by the use of existing reliable meshing tools. The other benefits of nodal-integration-based FEM come from the fact that all the mechanical 
quantities (displacements, strains, stresses, internal variables) are now defined at nodes. So the results files are much smaller than those coming from the classical FEM. In addition, post-processing is easier as it does not need an averaging procedure to extrapolate stresses and internal variables from Gauss points to the nodes. An easier transfer of mechanical quantities between two meshes is also expected. The main drawback of the method comes from a somewhat larger bandwidth of the tangent matrix. This drawback is compensated by the fact that the material constitutive equations are now solved at nodes that are much less numerous than Gauss points.

\section{ACKNOWLEDGEMENTS}

The authors wish to thanks China Scholarship Council (grant number 201608070111) for the financial support and Yonggang DUAN (ESI Group) for technique support.

\section{REFERENCES}

[1] Zienkiewicz, O.C. and Taylor, R.L. The finite element method. McGraw Hill, Vol. I., (1989), Vol.II., (1991)

[2] Bathe K., Finite Element Procedures, Prentice-Hall, Englewood Cliffs, 1996.

[3] Feulvarch, E., Amin-El-Sayed, H., Roux, J.-C., A stabilized P1/P1 finite element for the mechanical analysis of solid metals. Int J Mater Form 10, 314 (2017). https://doi.org/10.1007/s12289-015-
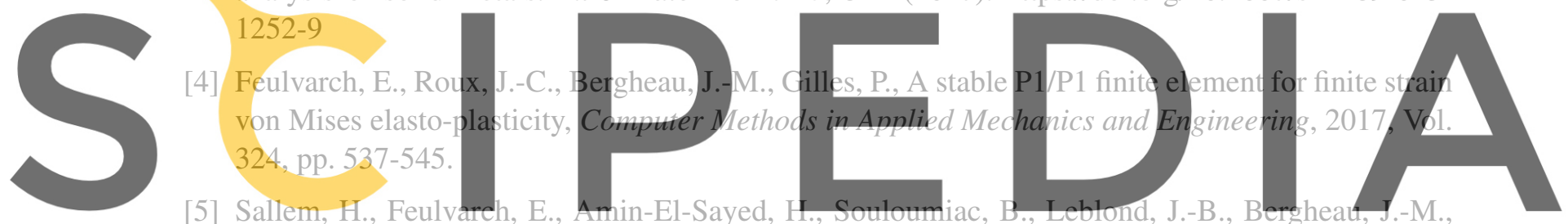

Recent advances in residual stress simulation caused by the welding process, COMPLAS XIII :

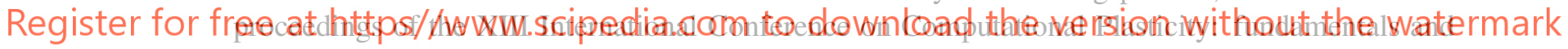
applications". CIMNE ed. Barcelona: CIMNE, 2015, p. 771-782.

[6] Arnold, D.N., Brezzi, F., Fortin, M., A stable finite element for the Stokes equations, Calcolo 1984;21:337344, doi.org/10.1007/BF02576171.

[7] Heuze, T., Amin-El-Sayed, H., Leblond, J.-B., Bergheau, J.-M.(2014), Benchmark tests based on the couette viscometerII: Thermo-elasto-plastic solid behaviour in small and large strains, Computers and Mathematics with Applications, 67(8), 1482-1496. doi:10.1016/j.camwa.2014.02.010.

[8] Chen, J.S., Wu, C.T., Yoon, S. and You, Y. (2001), A stabilized conforming nodal integration for Galerkin meshfree methods. Int. J. Numer. Meth. Engng., 50: 435-466. https://doi.org/10.1002/1097-0207(20010120)50:2;435::AID-NME32;3.0.CO;2-A

[9] Chen, J.S., Yoon, S. and Wu, C.T. (2002), Nonlinear version of stabilized conforming nodal integration for Galerkin meshfree methods. Int. J. Numer. Meth. Engng., 53: 2587-2615. https://doi.org/10.1002/nme.338

[10] William Elmer, J.S. Chen, Mike Puso, Ertugrul Taciroglu. A stable, meshfree, nodal integration 
method for nearly incompressible solids. Finite Element in Analysis and Design, 2012; 51:8185. https://doi.org/10.1016/j.finel.2011.11.001

[11] M. A. Puso and J. Solberg (2006), A stabilized nodally integrated tetrahedral, Int. J. Numer. Meth. Eng., 67: 841-867. https://doi.org/10.1002/nme.1651

[12] Bonet, J. and Burton, A.J. (1998), A simple average nodal pressure tetrahedral element for incompressible and nearly incompressible dynamic explicit applications. Commun. $\mathrm{Nu}$ mer. Meth. Engng., 14: 437-449. https://doi.org/10.1002/(SICI)1099-0887(199805)14:5¡437::AIDCNM162;3.0.CO;2-W

[13] Dohrmann, C., Heinstein, M.W., Jung, J., Key, S.W. and Witkowski, W.R. (2000), Nodebased uniform strain elements for threenode triangular and fournode tetrahedral meshes. Int. J. Numer. Meth. Engng., 47: 1549-1568. https://doi.org/10.1002/(SICI)1097-0207(20000330)47:9;1549::AIDNME842 ¿3.0.CO;2-K

[14] Bonet, J., Marriott, H. and Hassan, O. (2001), An averaged nodal deformation gradient linear tetrahedral element for large strain explicit dynamic applications. Commun. Numer. Meth. Engng., 17: 551-561. https://doi.org/10.1002/cnm.429

[15] Krysi, P. and Zhu, B. (2008), Lockingfree continuum displacement finite elements with nodal integration. Int. J. Numer. Meth. Engng., 76: 1020-1043. https://doi.org/10.1002/nme.2354

[16] Quak, W., van den Boogaard, A.H., Gonzalez, D., Cueto, E., A comparative study on the performance of meshless approximations and their integration. Comput.Mech., 2011, 48:121137 DOI
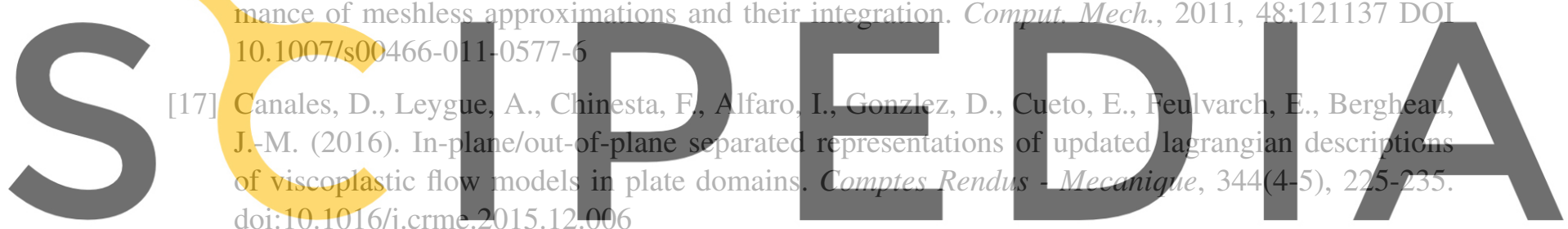

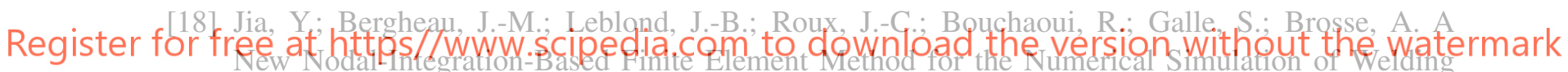
Processes. Metals 2020, 10, 1386.

[19] Software SYSWELD ${ }^{\mathrm{TM}}$ version 21, Reference Analysis Manual, Released: 2019; ESI-Group: France, Lyon, 2019. 